\title{
PROVIDING INFORMATION IN PUBLIC OPINION SURVEYS: MOTIVATION AND ABILITY EFFECTS IN THE INFORMATION-AND-CHOICE QUESTIONNAIRE
}

\section{Daan van Knippenberg and Dancker Daamen}

\begin{abstract}
The Information-and-Choice Questionnaire (ICQ) is an instrument for public opinion survey in which respondents are presented with a policy-relevant decision problem and provided with information relevant to the problem (e.g. information about the consequences of the policy options). In the present paper we study the effects of respondents' motivation (operationalized as involvement in the issue) and ability (operationalized as respondent's level of education) on the formation and stability of preferences in the ICQ. Data were gathered in a nation-wide $(N=99 \mathrm{I})$ survey on preferences for different ways of generating electricity in the Netherlands. As predicted, more motivated and more able respondents appeared to engage in more elaborate information processing.
\end{abstract}

Opinion polls may be useful tools to assess to what extent the public supports certain policies and to provide policy makers with information about public opinion. Yet, traditional public opinion surveys often yield information that policy makers may find problematic to incorporate into their decisions. Essentially, the information provided by traditional opinion surveys may be problematic for two reasons. First, whereas policy makers are usually faced with the choice between several alternative courses of action, opinion surveys often assess opinions about each separate alternative without actually requesting respondents to make a choice between alternatives. Second, opinion survey respondents may express opinions based on little or no knowledge of the policy issue (cf. Converse I964). As a consequence, policy makers may feel reluctant to incorporate opinion

The authors wish to express their thanks to Peter Neijens for his useful comments on the design of the ICQ and on a previous draft of this paper. 
survey results into their decision to the extent that they suspect that these opinions are based on insufficient knowledge of the policy issue.

Based on the work of Neijens (1987; Neijens et al. 1992) we designed a questionnaire that meets these problems. First, the policy issue was presented as a decision problem. Second, respondents were provided with information concerning the decision problem, the policy options available to the government and the consequences of the alternatives. The design of this questionnaire, called the Information-and-Choice Questionnaire (ICQ), is extensively discussed elsewhere (see Neijens 1987). Therefore, we will only discuss it briefly here.

\section{INFORMATION-AND-CHOICE QUESTIONNAIRE: POTENTIAL AND PROBLEMS}

The ICQ was originally developed by Saris, Neijens and De Ridder (see e.g. Neijens 1987, Neijens et al. 1992) to assess preferences for different ways of generating electricity in the Netherlands (but has since been used to assess preferences in other areas as well; see Alcser et al. in preparation, Neijens and de Ridder 1992, Neijens et al. 1996). The aim of the ICQ is not only to provide respondents with the necessary information to reach an informed opinion, but also to help them make use of this information to form opinions about different policy options: part of its aim is to guide respondents' information processing. Before respondents in the ICQ choose between policy options, they receive information to make a more informed choice. First, the choice is explicitly framed as a decision problem and respondents are informed about the background of the decision problem (e.g. they are told why these specific options are included in the decision problem). Second, respondents are provided with information about the consequences of the different policy options. To stimulate information processing and to help respondents reach a decision, they are requested to give a quantitative evaluation of each consequence. On the basis of these quantitative evaluations, the subjective utility of each option may be determined by summing the evaluations of the consequences of the option (cf. Edwards and Newman 1982). If respondents base their choices on these evaluations of consequences, they will choose the alternative(s) with the highest subjective utility (Neijens 1987 , Neijens et al. 1992). The ICQ procedure does, however, neither require nor request that respondents base their choices on these quantitative operationalizations of subjective utility.

The effects and usefulness of the ICQ have been studied in extensive evaluation research (Neijens 1987 ). Neijens shows that nonresponse in the ICQ is not substantially different from nonresponse in traditional opinion surveys (nonresponse is low and the group of nonrespondents has the same profile as the group that does respond) and concludes that the ICQ may be used to collect 
opinions of representative samples of the general public. In addition, Neijens found that preferences of respondents in an ICQ survey differ from those in a traditional survey, i.e. ICQ respondents make different choices than respondents in a survey in which no information about the policy options is provided. Van der Salm, van Knippenberg and Daamen (1995) provide experimental evidence for the fact that ICQ respondents' preferences are affected by the information provided in the ICQ. Neijens' examination of the correspondence between evaluations of consequences of the options and choices suggests that ICQ respondents tend to base their choices-at least in part-on their evaluations of the consequences of the options. Moreover, comparison of evaluation-choice correspondence in the ICQ with evaluation-choice correspondence in a survey in which respondents first made their choice and then evaluated the consequences of the options shows that respondents' choices correspond more to their evaluations in the ICQ (Neijens et al. 1992). This suggests that the ICQ's effect on respondents' preferences is probably due to both the information provided-which may wholly or in part contain new information relevant to the decision problem-and to better integration of the available information (due to the ICQ's structuring of information processing). The fact that ICQ respondents may report different preferences than respondents in a more traditional survey shows that it may indeed be worth the trouble to use the ICQ in public opinion research. At the same time it implies that the results of an ICQ do not necessarily reflect present public support for a policy. Rather the ICQ is especially suited to assess how public opinion may be after the public is informed about an issue or to assess the potential (i.e. after extra information is provided to the public) support for alternative policies.

The present study aims to evaluate the ICQ's potential for collecting informed public opinions by focusing on the effects of two factors essential to information processing and opinion formation: motivation and ability. Probably the main problem with any attempt to provide information in a public opinion survey is that it may fail as a consequence of insufficient motivation or ability to process the presented information on the respondents' part. The less motivated or able respondents are, the more likely it is that their opinions, preferences, and choices will not be based on the information provided to them. All other things being equal, more motivated respondents should be more likely to engage in systematic processing of provided information (that is, more extensively think about the issue-relevant arguments provided; cf. Petty and Cacioppo ig86). Similarly, all other things being equal, more able respondents should be more likely to systematically process information (cf. Chaiken 1980, 1987, Petty and Cacioppo 198r, 1986).

Respondents may only be expected to systematically process the presented information if they are sufficiently motivated and able to do so. The first concern 
in any attempt to give respondents information about a certain issue in the course of an opinion survey should be to minimize the chances that respondents will not be motivated or able to scrutinize the information provided (e.g. by ensuring that it does not take too long to complete the survey and by using simple wording). Yet, even when we take all necessary precautions, some respondents will still be more motivated (e.g. because of a specific interest or involvement in the issue) or more able (e.g. because of higher education) than others to process the information. The first question that will be addressed in this study is what influence motivation and ability exert on respondents' preference formation in the ICQ.

Ideally, if ICQ respondents systematically process the presented information, they should carefully evaluate each consequence, form an overall impression of each option based-at least in part-on active integration of the available information and base their final choice on their informed opinion about each alternative. Although it is impossible to assess exactly what respondents do while they fill in the ICQ we may get some indication of the extent to which respondents engage in systematic processing by focusing on the relationship between their evaluation of the consequences of the options and their choices. The better respondents process the information about the consequences of the different options, the more likely it is that their final decision will correspond to their evaluation of these consequences (cf. Petty and Cacioppo 1986). As a consequence, the correspondence between evaluations and choices may be taken to be indicative of the degree to which choices are based on elaborate processing of the information provided (Neijens I987, Neijens et al. 1992). We expect that more motivated respondents, as well as more able respondents, will engage in more extensive processing of the provided information. As a result, more motivated respondents' choices, as well as those of the more able, should correspond more to the evaluations of the consequences of the different options than less motivated and less able respondents' choices.

An additional indication of the elaborateness of respondents' information processing may be found in the stability of preferences over time. When preferences are based on extensive elaboration of information, they are more likely to be stable in time (cf. Petty and Cacioppo I986). So, if more motivated and more able respondents are more likely to engage in extensive elaboration of the information, their preferences should be more stable in time.

It seems reasonable to assume that motivation and ability do affect the degree to which respondents use the provided information. Yet, even if more motivated and more able respondents make better use of the information provided, it would seem important to establish to what extent the ICQ is able to inform less motivated and less able respondents. The possibility that more motivated and more able respondents make better use of the information provided does 
not preclude the possibility that less motivated and less able respondents use the information provided and are affected by the ICQ. Therefore, the second point we will address is the relationship between motivation and ability on the one hand and the extent to which respondents appear to be affected by the ICQ on the other.

\section{METHOD}

The ICQ reported here may be considered to be an update of the first ICQ (see Neijens 1987). It concerned the energy supply in the near future and was conducted by commission of the Dutch government. A representative sample of $99 \mathrm{I}$ respondents of $\mathrm{I} 8$ years and older from the Dutch public recruited from all over The Netherlands participated in the survey (December 1993-January 1994). These respondents were visited at their own home by professional interviewers using CAPI procedures. In an extensive introduction the ICQ procedures were explained and the decision problem was introduced, the choice of two out of six options to ensure the future supply of electricity. These options, which all provide an equal amount of electricity, are the use of traditional coal-fired plants, the use of coal-fired plants with removal of most $\mathrm{CO}_{2}$ (an innovative technique which has less harmful environmental consequences), the use of natural gas-fired plants, the use of nuclear power, a package of energy conservation measures, and an additional package of very strict conservation measures. Due to policy restrictions, not all of the possible combinations of these six options could be chosen, nor was it possible to choose options more than once. As a consequence, respondents could choose one out of ten possible combinations of two options. ${ }^{1}$

Before respondents made their choice, they received information (which they could read at their own pace) about the decision problem and the consequences of each of the six options. This information was compiled by various energy experts and translated for lay people by the researchers. It turned out that the experts agreed on most information about the consequences. If they disagreed, information about disagreement between experts was provided following Neijens ( 1987 ). This was the case where the potential effect of the use of nuclear energy on the proliferation of nuclear arms was concerned: respondents were informed that most experts did not think that such an effect would exist, while some experts thought that it would. As a safeguard against the use of incorrect or one-sided information, the validity of the information and the decision problem

\footnotetext{
1 These combinations were coal and natural gas, coal and nuclear energy, coal and conservation, coal with removal of $\mathrm{CO}_{2}$ and natural gas, coal with removal of $\mathrm{CO}_{2}$ and nuclear energy, coal with removal of $\mathrm{CO}_{1}$ and conservation, natural gas and nuclear energy, natural gas and conservation, nuclear energy and conservation, and a double package of conservation.
} 
was judged by an independent committee and only used after the committee's approval. The information concerned those consequences energy experts deemed the most important and the most relevant to the decision problem. An example of the kind of consequences that were described is provided in the Appendix, which contains the consequences of a choice for extra coal-fired plants. This information concerns various issues like the safety of plants, environmental concerns (the greenhouse effect, acid rain and coal waste) and fuel stocks. The information is not exhaustive: it only describes the consequences that were rank ordered by energy experts as the most important. Information concerning the consequences of the other options consisted of similar information.

The consequences were described on a separate showcard for each option. On these cards the consequences were described on the left hand side, while to the right respondents wrote down their evaluation of the consequence. Respondents were asked to indicate to what extent they thought each consequence was disadvantageous $(-9$, extreme disadvantage, to $-I$, slight disadvantage) or advantageous $(+1$, slight advantage, to +9 , extreme advantage) or irrelevant (o). A CAPI procedure provided respondents with feedback about the sum total of both the total perceived advantage and the total perceived disadvantage of each option (cf. Neijens 1987). These totals were also written down by the interviewer at the bottom of each showcard. Before respondents started with the actual ICQ they had practised with the response scales (ratings of utility and the sum totals based on these utility ratings) and the ICQ procedures (by means of a practice ICQ about a simple and fictitious unrelated decision problem).

If respondents based their choice on their evaluation of the consequences (and the evaluative ratings correctly represented respondents' evaluations), we would expect choices to correspond to utility scores. To assess evaluation-choice correspondence, the subjective utilities of the ten possible combinations of options were computed for each respondent (by summing the utility scores of the two options comprising the combination), and the ten combinations were rank ordered on the basis of these summed utilities. On the basis of this rank ordering we assigned the respondent's chosen combination a score between I, combination chosen has highest utility, and Io, combination chosen has lowest utility. This score reflects the extent to which the respondent's choice corresponds to the respondent's evaluation of the consequences of each option and may be taken to be indicative of the degree to which a respondent's choice is based on careful evaluation of the consequences of each option (cf. Neijens 1987, Neijens et al. 1992).

As an indication of respondents' motivation to process the information, involvement in the issue was assessed using a slightly modified version of Verplanken's (199I) scale for involvement with the energy issue. Issue in- 
volvement may be taken to be a good indicator of respondents' motivation to systematically process the presented information (cf. Petty and Cacioppo 1979, Petty et al. 198I). On the basis of a median split of the scores on the involvement scale a high involvement and a low involvement group were created. Because not all respondents completed the involvement scale, the final analysis was performed with $N=984$. Respondents' level of education was assessed as an indicator of respondents' ability to process the presented information (cf. Krosnick and Alwin 1987). Education was categorized as either high, medium or low.

Two months after the ICQ-survey ( $\mathrm{T}_{\mathrm{I}}$ ), a sample of 202 respondents from the original sample completed a second questionnaire $\left(\mathrm{T}_{2}\right)$. They were confronted with the same decision problem, but were not given any information about the consequences of the different policy options. Comparison of the preferences on $T_{1}$ with preferences on $T_{2}$ allows us to test the stability of preferences of ICQ respondents.

To determine the extent to which the ICQ appeared to affect respondents' preferences, respondents rated the extent to which they felt their opinion about the different ways of generating electricity had changed and the extent to which the way they thought about the different ways of generating electricity had changed in general. The average of these two ratings was computed as an indication of the ICQ's effect on respondents' opinions (self-reported effect; 7-point scale with higher scores indicating a greater effect on opinions).

\section{RESULTS}

\section{Evaluation-Choice Correspondence}

Table I provides, for each involvement and education group, an overview of the evaluation-choice correspondence. The degree to which respondents' choice corresponded to their evaluation of the consequences of the options, operationalized by the position of the chosen combination in the rank ordering of subjective utilities, was analyzed in an Involvement $\times$ Education ANOVA. Evaluation-choice correspondence was higher among the high involvement respondents $(M=2.32)$ than among the low involvement group $(M=2.79$; $F(\mathrm{I}, 978)=7.45, p<.0 \mathrm{I}$; lower scores indicate higher correspondence). Education and correspondence were also related $(F(1,978)=11.18, p<.0001)$. Evaluationchoice correspondence was stronger among higher educated respondents (low: $M=3.02$; medium: $M=2.74$; high: $M=2.16$ ). Obviously, motivation and ability affect respondents' preference formation as predicted. These results suggest that both more motivated and more able respondents tend to engage in more elaborate processing of the information presented. 
TABLE I Evaluation-choice correspondence as a function of involvement and education

\begin{tabular}{|c|c|c|c|c|c|}
\hline \multirow{2}{*}{$\begin{array}{l}\text { Rank order of } \\
\text { chosen } \\
\text { combination } \\
\text { according to } \\
\text { utility scores }\end{array}$} & \multicolumn{2}{|c|}{ Involvement } & \multicolumn{3}{|c|}{ Education } \\
\hline & Low & High & $\begin{array}{c}\text { Low } \\
\text { (Percent) }\end{array}$ & Medium & High \\
\hline I & $47 . I$ & 53.8 & $4^{6.2}$ & 47.8 & 54.8 \\
\hline 2 & 13.9 & I6.9 & 10.0 & 14.6 & 18.9 \\
\hline 3 & II.O & 8.6 & II.3 & 10.4 & 8.6 \\
\hline 4 & 6.1 & 6.3 & 6.3 & 5.9 & 6.4 \\
\hline 5 & $7 \cdot 3$ & 4.2 & 6.3 & 6.7 & 4.7 \\
\hline 6 & 4.9 & 4.6 & 7.7 & $5 \cdot 3$ & 2.7 \\
\hline 7 & 2.9 & I. 5 & 4. I & 2.5 & 1.0 \\
\hline 8 & 3.1 & 2.5 & $4 \cdot 5$ & $3 \cdot 4$ & 1.5 \\
\hline 9 & 2.2 & .8 & 2.3 & 1.7 & 1.0 \\
\hline 10 & I. 6 & .6 & I.4 & 1.7 & .5 \\
\hline Total & I00 & 100 & 100 & 100 & 100 \\
\hline
\end{tabular}

Note: Entries show the percentage of respondents who chose a particular combination of options. Combinations were rank ordered according to subjective utility scores; e.g. 47.I percent in the low involvement group chose the combination of options that was most advantageous to them as indicated by subjective utility scores.

\section{Stability of Preferences}

By comparing choices on $\mathrm{T}_{1}$ with choices on $\mathrm{T}_{2}$, stability scores were computed for the 202 respondents who participated at $\mathrm{T}_{2}$. These scores range from $\mathrm{o}$, no change/stable preferences, to 2, complete change/two different options chosen/ unstable preferences. Stability scores were analyzed in an Involvement $\times$ Education ANOVA. We expected that more involved and better educated respondents' preferences would be more stable. As expected, stability was affected by both involvement and education. Higher education was associated with more stable preferences (low: $M=1.13$; medium: $M=.77$; high: $M=$ $.5 \mathrm{I} ; F(1,196)=9.09, p<.0001)$. This relation was moderated by respondents' involvement $(F(\mathrm{I}, 196)=3.27, p<.05$, for the Involvement $\times$ Education interaction). The education effect was stronger for low involvement respondents (low: $M=1.21$; medium: $M=.92$; high: $M=.32$ ) than for the high involvement group (low: $M=.91$; medium: $M=.67$; high: $M=.59$ ). This moderating effect of involvement was probably due to a stronger tendency to elaborately process the information among high involvement respondents, who were therefore more likely to reach stable preferences anyway. Again, results are in line with the 
prediction that more motivated and more able respondents are more likely to engage in extensive information processing.

\section{Self-Reported EFfect}

Respondents' ratings of the ICQ's effects on their opinions were analyzed in an Involvement $\times$ Education ANOVA. Low education respondents reported a greater effect (low: $M=3.67$; medium: $M=2.97$; high: $M=2.90 ; F(\mathrm{I}, 978)=$ I $4.77, p<.0001$ ). This effect was stronger for the high involvement group (low: $M=4.16$; medium: $M=2.83$; high: $M=2.73$ ) than for the low involvement group (low: $M=3.37$; medium: $M=3.09$; high: $M=3.10 ; F(1,978)=8.52$, $p<.000 \mathrm{I}$, for the Involvement $\times$ Education interaction). That is, less educated respondents reported more effect from the ICQ especially when their involvement in the issue is high.

For exploratory purposes we examined the relationship between the selfreported effect on the one hand and the evaluation-choice correspondence and stability on the other. Both relationships were weak (self-reported effect and correspondence: $r=-.03, n s$; ; self-reported effect and stability: $r=.12, p<.05$. We also tested the effects of involvement and education on the self-reported effect and correspondence, as well as on the self-reported effect and stability relationships, using a test of interactions in regression analysis. None of the interactions was significant. Apparently, the degree to which respondents systematically process the presented information is mostly unrelated to the degree to which they claim to be affected by the ICQ.

\section{DISCUSSION}

Motivation and ability affect decision making in the ICQ as expected: the analysis of the evaluation - choice correspondence suggests that more motivated and more able respondents engage in more elaborate information processing. Although we have no data on respondents' actual thoughts, the fact that more motivated and more able respondents' choices corresponded more to their evaluations suggests that these respondents thought more extensively about the presented information and integrated the relevant information more effectively into their final decision. These results may be considered to corroborate conclusions from studies on motivation and ability effects on the processing of persuasive communication (see e.g. Chaiken and Stangor 1987, Petty and Cacioppo 1986, Eagly and Chaiken I993).

Results of the analysis of the data on the stability of preferences seem also 
to suggest that more able and more motivated ICQ respondents engage in more elaborate processing. In addition, the stability data corroborate the results of studies showing that more elaborate processing tends to result in more stable preferences (Chaiken 1980, Chaiken and Eagly 1983, Mackie 1987). Yet, the stability results reported in the present paper may just as easily be explained as direct effects of involvement and education (i.e. more involved and better educated people being more stable in their preferences regardless of the ICQ) rather than as indications of more elaborate processing. For instance, it is possible that better educated people are generally better informed about policy issues and hold more informed opinions than less educated people, as a consequence of which their preferences, once formed, are more stable anyway. Yet, given the results for the evaluation-choice correspondence, we are inclined to conclude that more involved and higher educated respondents are more likely to engage in systematic processing of the information provided in the ICQ.

We should be cautious about what to conclude on the basis of the self-reported effect measure. First, people reporting a greater effect may simply be less reluctant to admit being affected rather than actually experiencing a greater effect. Second, people may simply be mistaken in their assessment of the degree to which they changed their opinions about a topic. Nevertheless, it is still interesting to note that less educated respondents claim a greater effect for the ICQ. This may corroborate Neijens' (1987) notion that the ICQ is a useful instrument for collecting informed public opinions among representative samples of the population. Interestingly, the people reporting a greater effect of the ICQ (i.e. less educated respondents) actually appear to have engaged in less systematic processing. The result is in line with results of a study by Eagly and Warren (1976), who found that more intelligent respondents are more likely to comprehend a message, while at the same time they may be less likely to change their attitudes in reaction to the message (i.e. systematic processing does not necessarily result in attitude change; cf. Petty and Cacioppo 1986, Chaiken 1987).

The results of the present study also give rise to a word of warning concerning the design of an ICQ: we should take care not to make an ICQ too taxing on respondents' motivation or ability. Only if the issue at hand is sufficiently involving for the majority of the respondents and only if we are able to present all necessary information without resorting to large amounts of very complex information, will it be possible to collect informed public opinions. In this case the use of the ICQ may provide policy makers with valuable information about how the public opinion, or public support for a given policy, will be once the public is properly informed about the consequences of certain policies. Given the fact that the implementation of new government policy is nowadays often accompanied by large information campaigns, it would seem that the use of the ICQ should be increasingly worthwhile in public opinion research. 


\section{APPENDIX \\ CONSEQUENCES OF THE USE OF EXTRA COAL-FIRED PLANTS ${ }^{2}$}

\section{Safety of Plants}

If an accident with a coal-fired plant occurs, people living in the neighborhood of the plant may, in the worst case, suffer from irritated eyes or bronchial tubes. It is virtually impossible that people living in the neighborhood will die or get injured if such an accident occurs. If such an accident occurs, personnel from the plant may die. Choice for 14 units additional coal results in a small chance of an accident as described above.

\section{SafETy of Extraction and Transport}

The extraction and transport of coal results in certain risks like health problems for miners and the risk of mining accidents. As a consequence of a choice for i4 units additional coal, a few deaths a year may be expected due to these extraction and transport risks.

\section{Contribution to Acid Rain}

The generation of electricity from coal contributes to acid rain. In the Netherlands, acid rain may result in the extinction of species of animals and plants in nature reserves, death of forests, damage to agriculture, to monuments and to properties, and to heathlands and a deterioration of drinking-water sources. At the moment these effects exist already to a lesser or larger degree. The contribution to acid rain effects of 14 units additional coal is about equal to the contribution of $\mathrm{I} 4$ units 'coal with removal of $\mathrm{CO}_{2}$ ' and twice as large as the contribution of 14 units natural gas. Compared to plants already under construction or in operation 14 units additional coal will enlarge the contribution of the electricity supply to the acid rain effects by about 8 percent.

\section{Contribution to the Greenhouse Effect}

The generation of electricity from coal contributes to the greenhouse effect, As a result of this effect, the average temperature on earth will probably increase in the long run. If the extent of the greenhouse effect is as predicted, a change of weather and climate may occur on a world-wide scale. This change may be so quick that it results in the extinction of certain species of animals and plants. It may also result in damage to agriculture (aridity, water damage or plagues of insects) and in extreme weather conditions (hurricanes and floods). The contribution of 14 units additional coal to the greenhouse effect is about twice as large as the contribution of ${ }_{4} 4$ units natural gas and much larger than the contribution of 14 units 'coal with removal of $\mathrm{CO}_{2}$ '. Compared to plants already under construction or in operation, 14 units additional cosal will enlarge the contribution of the electricity supply to the greenhouse effect by about 16 percent.

\section{Storage of Coal Waste}

The ash produced when generating electricity in coal-fired plants cannot al ways be recycled. As a consequence, part of the ash must probably be stored in the future for a shorter or longer period. Storage of coal-ash produces hardly any risk for the environment. But stored coal-ash may be conspicuous in the landscape. 14 units additional coal will enlarge the chances that coal ash must be stored.

\footnotetext{
${ }^{2}$ Options were presented as potential providers of a fixed amount of electricity: 14 'units'. These units were related to the present electricity supply.
} 


\section{Coal Stocks}

Expectations are that the coal stocks in the world will suffice for a few centuries. The use of 14 units additional coal to generate electricity will somewhat enlarge the pace of depletion of coal supplies in the world.

\section{REFERENCES}

Alcser, Kirsten H., Neijens, Peter C., and Bachman, Jerald G. (in preparation): 'Informed public opinion on euthanasia and doctor assisted suicide: A comparison between the state of Michigan and the Netherlands'.

Chaiken, Shelly (1980): 'Heuristic versus systematic information processing and the use of source versus message cues in persuasion', Foumal of Personality and Social Psychology, 39, 752-66.

Chaiken, Shelly (1987): 'The heuristic model of persuasion'. In M. P. Zanna, J. M. Olsen and C. P. Herman (eds.) Social infuence: The Ontario symposium, Vol. 5, Hillsdale, NJ, Erlbaum.

Chaiken, Shelly and Eagly, Alice H. (1983): 'Communication modality as a determinant of persuasion: The role of communicator salience,' Journal of Personality and Social Psychology, 34, 605-I4.

Chaiken, Shelly and Stangor, Charles (1987): 'Attitudes and attitude change', Annual Review of Psychology, 38, 575-630.

Converse, Philip E. (1964): 'The nature of belief systems in mass publics.' In D. E. Apter (ed.) Ideology and discontent, New York, Free Press.

Eagly, Alice H. and Chaiken, Shelly (1993): The Psychology of Attitudes, Orlando, FL, Harcourt Brace Jovanovich.

Eagly, Alice H. and Warren, Rebecca (1976): 'Intelligence, comprehension, and opinion change,' Journal of Personality, 44, 226-42.

Edwards, Ward and Newman, J. Robert (1982): Multiattribute Evaluation, Beverly Hills/London, Sage.

Krosnick, Jon A. and Alwin, Duane F. (1987): 'An evaluation of a cognitive theory of response-order effects in survey measurement,' Public Opinion Quarterly, 5I, 20I-19.

Mackie, Diane M. (1987): 'Systematic and nonsystematic processing of majority and minority persuasive communications,' Journal of Personality and Social Psychology, $53,4^{1-52}$.

Neijens, Peter (1987): The Choice Questionnaire. Design and Evaluation of an Instrument for Collecting Informed Opinions of a Population, Amsterdam, Free University Press.

Neijens, Peter C. and De Ridder, Jan A. (1992): 'De keuze-enquête over de auto in de binnenstad.' In W. E. Saris, P. C. Neijens and J. J. M. Slot (eds.) Het Amsterdamse referendum in perspectief, Amsterdam, Cramwinckel.

Neijens, Peter, De Ridder, Jan A. and Saris, Willem E. (1992): 'An instrument for collecting informed opinions,' Quality and Quantity, 26, 245-58.

Neijens, Peter, Minkman, Mark, de Ridder, Jan, Saris, Willem and Slot, Jeroen (1996): 'A decision aid in a referendum,' International foumal of Public Opinion Research, 8 (in this issue). 
Petty, Richard E. and Cacioppo, John T. (1979): 'Issue involvement can increase or decrease persuasion by enhancing message-relevant cognitive responses,' Journal of Personality and Social Psychology, 37, 1915-26.

Petty, Richard E. and Cacioppo, John T. (1981): Attitudes and Persuasion: Classic and Contemporany Approaches, Dubuque, Iowa, W. C. Brown.

Petty, Richard E. and Cacioppo, John T. (1986): 'The elaboration likelihood model of persuasion.' In L. Berkowitz (ed.) Advances in Experimental Social Psychology, Vol. 19, New York, Academic Press.

Petty, Richard E., Cacioppo, John T. and Goldman, Rachel (198I): 'Personal involvement as a determinant of argument-based persuasion,' foumal of Personality and Social Psychology, 4I, 847-55.

van der Salm, Corinne A., van Knippenberg, Daan and Daamen, Dancker D. L. (1995): 'A critical test of the choice questionnaire for collecting informed public opinions.' Manuscript submitted for publication.

Verplanken, Bas (199I): 'Persuasive communication of risk information: A test of cue versus message processing effects in a field experiment,' Personality and Social Psychology Bulletin, 17, 188-93.

\section{BIOGRAPHICAL NOTES}

At the time of the survey, Daan van Knippenberg was a researcher at the Centre for Energy and Environmental Research, University of Leiden. At present he is an assistant professor at the Department of Social and Organizational Psychology, University of Leiden.

Dancker Daamen is a senior researcher at the Centre for Energy and Environmental Research and an assistant professor at the Department of Social and Organizational Psychology, University of Leiden. 